\title{
NON-EXTENDABILITY OF THE FINITE HILBERT TRANSFORM
}

\author{
GUILLERMO P. CURBERA, SUSUMU OKADA, AND WERNER J. RICKER
}

\begin{abstract}
The finite Hilbert transform $T: X \rightarrow X$ acts continuously on every rearrangement invariant space $X$ on $(-1,1)$ having non-trivial Boyd indices. It is proved that $T$ cannot be further extended, whilst still taking its values in $X$, to any larger domain space. That is, $T: X \rightarrow X$ is already optimally defined.
\end{abstract}

\section{INTRODUCTION AND MAIN RESULT}

The finite Hilbert transform $T(f)$ of $f \in L^{1}(-1,1)$ is the well known principal value integral

$$
(T(f))(t)=\lim _{\varepsilon \rightarrow 0^{+}} \frac{1}{\pi}\left(\int_{-1}^{t-\varepsilon}+\int_{t+\varepsilon}^{1}\right) \frac{f(x)}{x-t} d x,
$$

which exists for a.e. $t \in(-1,1)$ and is a measurable function. It has important applications to aerodynamics and elasticity via the airfoil equation, [3], [16, [20, [23], 24], and to problems arising in image reconstruction; see, for example, [11], [21]. We refer to [7], [8], [9], 10], [15] where one-dimensional singular integral operators closely related to the finite Hilbert transform are studied in great detail

For each $1<p<\infty$ the classical linear operator $f \mapsto T(f)$ maps $L^{p}(-1,1)$ continuously into itself; denote this operator by $T_{p}$. Tricomi showed that $T_{p}$ is a Fredholm operator and exhibited inversion formulae, [23], except for the case when $p=2,[24, \S 4.3$ ] (see also [12, Ch. 11], [19, Ch. 14.4-3] and the references therein). For $T_{2}$ the situation is significantly different, as already pointed out somewhat earlier in [22, p.44]. Partial operator theoretic results for $T_{2}$ on $L^{2}(-1,1)$ were obtained by Okada and Elliott, [17]; see also the references.

In [4] the finite Hilbert transform $T$ was studied when acting on suitable rearrangement invariant (r.i., in short) spaces $X$ on $(-1,1)$; see below for the relevant definitions. Actually, $T$ acts continuously on $X$ (denote this operator by $T_{X}$ ) precisely when the Boyd indices of $X$ are non-trivial, that is, when $0<\underline{\alpha}_{X} \leqslant \bar{\alpha}_{X}<1$; see [13, pp.170-171]. This class of r.i. spaces is the largest and most adequate replacement for the $L^{p}$-spaces when undertaking a further study of the finite Hilbert transform $T$. This is due to two

Date: May 14, 2021.

2010 Mathematics Subject Classification. Primary 44A15, 46E30; Secondary 47A53, 47B34.

Key words and phrases. Finite Hilbert transform, rearrangement invariant space, airfoil equation, Fredholm operator.

The first author acknowledges the support of of PGC2018-096504-B-C31, FQM-262 and Feder-US1254600 (Spain). 
critical facts: that $T: X \rightarrow X$ is injective if and only if the function $1 / \sqrt{1-x^{2}} \notin X$, and that $T: X \rightarrow X$ has non-dense range if and only if $1 / \sqrt{1-x^{2}}$ belongs to the associate space $X^{\prime}$ of $X$ (whenever $X$ is separable). In terms of r.i. spaces the previous conditions can be phrased as follows: $T: X \rightarrow X$ is injective if and only if $L^{2, \infty}(-1,1) \nsubseteq X$ and $T: X \rightarrow X$ has a non-dense range if and only if $X \subseteq L^{2,1}(-1,1)$ (for $X$ separable). Here $L^{2,1}(-1,1)$ and $L^{2, \infty}(-1,1)$ are the usual Lorentz spaces.

Various types of inversion results of Tricomi for the operator $T_{p}$ (when $1<p<2$ and $2<p<\infty$ ) have been extended to $T_{X}$ whenever the Boyd indices of $X$ satisfy the condition $0<\underline{\alpha}_{X} \leqslant \bar{\alpha}_{X}<1 / 2$ or $1 / 2<\underline{\alpha}_{X} \leqslant \bar{\alpha}_{X}<1$; see [4, Theorems 3.2 and 3.3]. Moreover, $T$ is necessarily a Fredholm operator in such r.i. spaces, [4, Remark 3.4]. Results of this kind admit the possibility for a refinement of the solution of the airfoil equation; see [4, Corollary 3.5]. Additional operator theoretic results concerning $T_{X}$ in r.i. spaces $X$ occur in the recent article [5] (e.g., compactness, order boundedness, integral representation, etc.).

An important problem is the possibility of extending the domain of $T_{p}$, with $T_{p}$ still maintaining its values in $L^{p}(-1,1)$. It was shown in [18, Example 4.21], for all $1<p<\infty$ with $p \neq 2$, that there is no larger Banach function space (B.f.s. in short) containing $L^{p}(-1,1)$ such that $T_{p}$ has an $L^{p}(-1,1)$-valued continuous extension to this space. This result was generalized in [4, Theorem 4.7]. Namely, it is not possible to extend the finite Hilbert transform $T_{X}: X \rightarrow X$ for any r.i. space $X$ satisfying

$$
0<\underline{\alpha}_{X} \leqslant \bar{\alpha}_{X}<1 / 2 \text { or } 1 / 2<\underline{\alpha}_{X} \leqslant \bar{\alpha}_{X}<1 \text {. }
$$

The arguments used in [4] for establishing the above result do not apply to $T_{X}$ for r.i. spaces $X$ which fail to satisfy (1). In particular, they do not apply to $T_{2}: L^{2}(-1,1) \rightarrow$ $L^{2}(-1,1)$. However, in [4] it was also established, via a completely different approach, that at least $T_{2}$ does not have a continuous $L^{2}(-1,1)$-valued extension to any larger B.f.s., [4, Theorem 5.3].

Thus, the question of extendability of $T_{X}$ remains unanswered for a large sub-family of r.i. spaces which have non-trivial Boyd indices. Indeed, with the exception of $X=$ $L^{2}(-1,1)$, this is the case for all those r.i. spaces $X$ satisfying $0<\underline{\alpha}_{X} \leqslant 1 / 2 \leqslant \bar{\alpha}_{X}<1$. In particular, this includes all the Lorentz spaces $L^{2, q}$ for $1 \leqslant q \leqslant \infty$ with $q \neq 2$. The proof given in [4] for $T_{2}$, based on the Hilbert space structure of $L^{2}(-1,1)$, is not applicable to other r.i. spaces of the kind just mentioned.

The aim of this note is to answer the above question for all r.i. spaces $X$ on which $T_{X}$ is continuous, via a new and unified proof.

Theorem. Let $X$ be a r.i. space on $(-1,1)$ with non-trivial Boyd indices. The finite Hilbert transform $T_{X}: X \rightarrow X$ has no continuous, $X$-valued extension to any genuinely larger B.f.s. containing $X$.

\section{Preliminaries}

In this paper the relevant measure space is $(-1,1)$ equipped with its Borel $\sigma$-algebra $\mathcal{B}$ and Lebesgue measure $m$ (restricted to $\mathcal{B}$ ). We denote by $L^{0}(-1,1)=L^{0}$ the space 
(of equivalence classes) of all $\mathbb{C}$-valued measurable functions, endowed with the topology of convergence in measure. The space $L^{p}(-1,1)$ is denoted simply by $L^{p}$, for $1 \leqslant p \leqslant \infty$.

A Banach function space (B.f.s.) $X$ on $(-1,1)$ is a Banach space $X \subseteq L^{0}$ satisfying the ideal property, that is, $g \in X$ and $\|g\|_{X} \leqslant\|f\|_{X}$ whenever $f \in X, g \in L^{0}$ and $|g| \leqslant|f|$ a.e. The associate space $X^{\prime}$ of $X$ consists of all $g \in L^{0}$ satisfying $\int_{-1}^{1}|f g|<\infty$, for every $f \in X$, equipped with the norm $\|g\|_{X^{\prime}}:=\sup \left\{\left|\int_{-1}^{1} f g\right|:\|f\|_{X} \leqslant 1\right\}$. The space $X^{\prime}$ is a closed subspace of the Banach space dual $X^{*}$ of $X$. The space $X$ satisfies the Fatou property if, whenever $\left\{f_{n}\right\}_{n=1}^{\infty} \subseteq X$ satisfies $0 \leqslant f_{n} \leqslant f_{n+1} \uparrow f$ a.e. with $\sup _{n}\left\|f_{n}\right\|_{X}<\infty$, then $f \in X$ and $\left\|f_{n}\right\|_{X} \rightarrow\|f\|_{X}$. In this paper all B.f.s.' $X$ are on $(-1,1)$ relative to Lebesgue measure and, as in [1, satisfy the Fatou property.

A rearrangement invariant (r.i.) space $X$ on $(-1,1)$ is a B.f.s. such that if $g^{*} \leqslant f^{*}$ with $f \in X$, then $g \in X$ and $\|g\|_{X} \leqslant\|f\|_{X}$. Here $f^{*}:[0,2] \rightarrow[0, \infty]$ is the decreasing rearrangement of $f$, that is, the right continuous inverse of its distribution function: $\lambda \mapsto m(\{t \in(-1,1):|f(t)|>\lambda\})$. The associate space $X^{\prime}$ of a r.i. space $X$ is again a r.i. space. Every r.i. space $X$ on $(-1,1)$ satisfies $L^{\infty} \subseteq X \subseteq L^{1}$. Moreover, if $f \in X$ and $g \in X^{\prime}$, then $f g \in L^{1}$ and $\|f g\|_{L^{1}} \leqslant\|f\|_{X}\|g\|_{X^{\prime}}$, i.e., Hölder's inequality is available.

The family of r.i. spaces includes many classical spaces appearing in analysis, in particular the Lorentz $L^{p, q}$ spaces, [1, Definition IV.4.1].

Given a r.i. space $X$ on $(-1,1)$, due to the Luxemburg representation theorem there exists a r.i. space $\tilde{X}$ on $(0,2)$ such that $\|f\|_{X}=\left\|f^{*}\right\|_{\tilde{X}}$ for $f \in X$, [1, Theorem II.4.10]. The dilation operator $E_{t}$ for $t>0$ is defined, for each $f \in \tilde{X}$, by $E_{t}(f)(s):=f(s t)$ for $0 \leqslant s \leqslant \min \{2,1 / t\}$ and zero for $\min \{2,1 / t\}<s \leqslant 2$. The operator $E_{t}: \tilde{X} \rightarrow \tilde{X}$ is bounded with $\left\|E_{1 / t}\right\|_{\tilde{X} \rightarrow \tilde{X}} \leqslant \max \{t, 1\}$. The lower and upper Boyd indices of $X$ are defined, respectively, by

$$
\underline{\alpha}_{X}:=\sup _{0<t<1} \frac{\log \left\|E_{1 / t}\right\|_{\tilde{X} \rightarrow \tilde{X}}}{\log t} \text { and } \bar{\alpha}_{X}:=\inf _{1<t<\infty} \frac{\log \left\|E_{1 / t}\right\|_{\tilde{X} \rightarrow \tilde{X}}}{\log t} ;
$$

see [2] and also [1, Definition III.5.12]. They satisfy $0 \leqslant \underline{\alpha}_{X} \leqslant \bar{\alpha}_{X} \leqslant 1$. Note that $\underline{\alpha}_{L^{p}}=\bar{\alpha}_{L^{p}}=1 / p$.

For all of the above and further facts on r.i. spaces see [1], for example.

\section{Proof of the Theorem}

Given $X$, a r.i. space on $(-1,1)$ with non-trivial Boyd indices, consider the space

$$
[T, X]:=\left\{f \in L^{1}: T(h) \in X, \forall|h| \leqslant|f|\right\},
$$

which is a B.f.s. for the norm

$$
\|f\|_{[T, X]}:=\sup _{|h| \leqslant|f|}\|T(h)\|_{X}, \quad f \in[T, X] .
$$

The proof of this fact uses, in an essential way, a deep result of Talagrand concerning $L^{0}$-valued measures, [4, Proposition 4.5]. The space $[T, X]$ is the largest B.f.s. containing $X$ to which $T_{X}: X \rightarrow X$ has a continuous, linear, $X$-valued extension, [4, Theorem 4.6]. 
In particular, $X \subseteq[T, X]$. Thus, in order to show that no genuine extension of $T_{X}$ is possible it suffices to show that $[T, X] \subseteq X$; see Theorems 4.7 and 5.3 in [4].

$\operatorname{Fix} N \in \mathbb{N}$. Given $a_{1}, \ldots, a_{N} \in \mathbb{C}$ and disjoint sets $A_{1} \ldots, A_{N}$ in $\mathcal{B}$, define the simple function

$$
\phi:=\sum_{n=1}^{N} a_{n} \chi_{A_{n}} .
$$

On $\Lambda:=\{1,-1\}^{N}$ consider the probability measure $d \sigma$, which is the product measure of $N$ copies of the uniform probability on $\{1,-1\}$. Define the bounded measurable function $F$ on $\Lambda$ by

$$
\sigma=\left(\sigma_{1}, \ldots, \sigma_{N}\right) \in \Lambda \mapsto F(\sigma):=\left\|T_{X}\left(\sum_{n=1}^{N} \sigma_{n} a_{n} \chi_{A_{n}}\right)\right\|_{X} .
$$

Observe, since the sets $A_{1}, \ldots, A_{N}$ are pairwise disjoint, that for every $\sigma=\left(\sigma_{1}, \ldots, \sigma_{N}\right) \in$ $\Lambda$ one has

$$
\left|\sum_{n=1}^{N} \sigma_{n} a_{n} \chi_{A_{n}}\right|=\sum_{n=1}^{N}\left|a_{n}\right| \chi_{A_{n}}=|\phi|
$$

whence

$$
\|F\|_{L^{\infty}(\Lambda)}=\sup _{\sigma \in \Lambda}\left\|T\left(\sum_{n=1}^{N} \sigma_{n} a_{n} \chi_{A_{n}}\right)\right\|_{X} \leqslant \sup _{|h| \leqslant|\phi|}\|T(h)\|_{X}=\|\phi\|_{[T, X]} .
$$

On the other hand, an application of Fubini's theorem yields

$$
\begin{aligned}
\|F\|_{L^{\infty}(\Lambda)} & \geqslant\|F\|_{L^{1}(\Lambda)} \\
& =\int_{\Lambda}|F(\sigma)| d \sigma \\
& =\int_{\Lambda}\left\|\sum_{n=1}^{N} \sigma_{n} a_{n} T\left(\chi_{A_{n}}\right)\right\|_{X} d \sigma \\
& =\int_{\Lambda}\left(\sup _{\|g\|_{X^{\prime}}=1} \int_{-1}^{1}|g(t)|\left|\sum_{n=1}^{N} \sigma_{n} a_{n} T\left(\chi_{A_{n}}\right)(t)\right| d t\right) d \sigma \\
& \geqslant \sup _{\|g\|_{X^{\prime}}=1} \int_{\Lambda}\left(\int_{-1}^{1}|g(t)|\left|\sum_{n=1}^{N} \sigma_{n} a_{n} T\left(\chi_{A_{n}}\right)(t)\right| d t\right) d \sigma \\
& =\sup _{\|g\|_{X^{\prime}}=1} \int_{-1}^{1}|g(t)|\left(\int_{\Lambda}\left|\sum_{n=1}^{N} \sigma_{n} a_{n} T\left(\chi_{A_{n}}\right)(t)\right| d \sigma\right) d t .
\end{aligned}
$$

Consider now the inner integral over $\Lambda$ in the last term (3) of the previous expression. For $t \in(-1,1)$ fixed, set

$$
\beta_{n}:=a_{n} T\left(\chi_{A_{n}}\right)(t), \quad n=1 \ldots, N .
$$


It is known that the coordinate projections

$$
P_{n}: \sigma \in \Lambda \mapsto \sigma_{n} \in\{-1,1\}, \quad n=1, \ldots, N,
$$

form an orthonormal set, that is,

$$
\int_{\Lambda} P_{j} P_{k} d \sigma=\int_{\Lambda} \sigma_{j} \sigma_{k} d \sigma=\delta_{j, k}, \quad j, k=1, \ldots, N
$$

Then, for the inner integral in (3), we have

$$
\int_{\Lambda}\left|\sum_{n=1}^{N} \sigma_{n} a_{n} T\left(\chi_{A_{n}}\right)(t)\right| d \sigma=\int_{\Lambda}\left|\sum_{n=1}^{N} \beta_{n} P_{n}(\sigma)\right| d \sigma
$$

Apply the Khintchine inequality, [6, Inequality 1.10 and p.23], for $\left\{P_{n}\right\}_{n=1}^{N}$ yields

$$
\int_{\Lambda}\left|\sum_{n=1}^{N} \beta_{n} P_{n}(\sigma)\right| d \sigma \geqslant \frac{1}{\sqrt{2}}\left(\sum_{n=1}^{N}\left|\beta_{n}\right|^{2}\right)^{1 / 2} \text {. }
$$

Accordingly,

$$
\int_{\Lambda}\left|\sum_{n=1}^{N} \sigma_{n} a_{n} T\left(\chi_{A_{n}}\right)(t)\right| d \sigma \geqslant \frac{1}{\sqrt{2}}\left(\sum_{n=1}^{N}\left|a_{n}\right|^{2}\left|T\left(\chi_{A_{n}}\right)(t)\right|^{2}\right)^{1 / 2} .
$$

Then, from (3) and (4), it follows that

$$
\begin{aligned}
\|F\|_{L^{\infty}(\Lambda)} & \geqslant \frac{1}{\sqrt{2}} \sup _{\|g\|_{X^{\prime}}=1} \int_{-1}^{1}|g(t)|\left(\sum_{n=1}^{N}\left|a_{n}\right|^{2}\left|T\left(\chi_{A_{n}}\right)(t)\right|^{2}\right)^{1 / 2} d t \\
& =\frac{1}{\sqrt{2}}\left\|\left(\sum_{n=1}^{N}\left|a_{n}\right|^{2}\left|T\left(\chi_{A_{n}}\right)\right|^{2}\right)^{1 / 2}\right\|_{X} \cdot
\end{aligned}
$$

We recall the following consequence of the Stein-Weiss formula for the distribution function of the Hilbert transform $H$ on $\mathbb{R}$ of a characteristic function, due to Laeng, [14, Theorem 1.2]. Namely, for $A \subseteq \mathbb{R}$ with $m(A)<\infty$ (where $m$ also denotes Lebesgue measure in $\mathbb{R}$ ), we have

$$
\left.m\left(\left\{x \in A: \mid H\left(\chi_{A}\right)(x)\right) \mid>\lambda\right\}\right)=\frac{2 m(A)}{e^{\pi \lambda}+1}, \quad \lambda>0 .
$$

In particular, for any set $A \subseteq(-1,1)$ it follows, for each $\lambda>0$, that

$$
m\left(\left\{x \in A:\left|T\left(\chi_{A}\right)(x)\right|>\lambda\right\}\right)=m\left(\left\{x \in A:\left|H\left(\chi_{A}\right)(x)\right|>\lambda\right\}\right)=\frac{2 m(A)}{e^{\pi \lambda}+1} .
$$

That is,

$$
m\left(\left\{x \in A:\left|T\left(\chi_{A}\right)(x)\right|>\lambda\right\}\right)=\frac{2 m(A)}{e^{\pi \lambda}+1}, \quad A \in \mathcal{B}, \quad \lambda>0 .
$$

Set $\lambda=1$ and $\delta:=2 /\left(e^{\pi}+1\right)<1$. For each $n=1, \ldots, N$, define

$$
A_{n}^{1}:=\left\{x \in A_{n}:\left|T\left(\chi_{A_{n}}\right)(x)\right|>1\right\} .
$$


Then (6) implies that

$$
m\left(A_{n}^{1}\right)=\frac{2 m\left(A_{n}\right)}{e^{\pi}+1}=\delta m\left(A_{n}\right), \quad n=1, \ldots, N .
$$

Since the sets $A_{1}, \ldots, A_{N}$ are pairwise disjoint, so are their subsets $A_{1}^{1}, \ldots, A_{N}^{1}$. Note that $\left|T\left(\chi_{A_{n}}\right)(x)\right|>1$ for $x \in A_{n}^{1}$, for $n=1, \ldots, N$. Thus, on $(-1,1)$ we have the pointwise estimates

$$
\begin{aligned}
\left(\sum_{n=1}^{N}\left|a_{n}\right|^{2}\left|T\left(\chi_{A_{n}}\right)\right|^{2}\right)^{1 / 2} & \geqslant\left(\sum_{n=1}^{N}\left|a_{n}\right|^{2}\left|T\left(\chi_{A_{n}}\right)\right|^{2} \chi_{A_{n}}\right)^{1 / 2} \\
& =\sum_{n=1}^{N}\left|a_{n}\right|\left|T\left(\chi_{A_{n}}\right)\right| \chi_{A_{n}} \\
& \geqslant \sum_{n=1}^{N}\left|a_{n}\right| \chi_{A_{n}^{1}} .
\end{aligned}
$$

Since $\|\cdot\|_{X}$ is a lattice norm, (8) yields

$$
\left\|\left(\sum_{n=1}^{N}\left|a_{n}\right|^{2}\left|T\left(\chi_{A_{n}}\right)\right|^{2}\right)^{1 / 2}\right\|_{X} \geqslant\left\|\sum_{n=1}^{N}\left|a_{n}\right| \chi_{A_{n}^{1}}\right\|_{X}=\|\varphi\|_{X},
$$

where $\varphi$ is the simple function

$$
\varphi:=\sum_{n=1}^{N} a_{n} \chi_{A_{n}^{1}}
$$

From (7) it follows that

$$
m(\{x \in(-1,1):|\varphi(x)|>\lambda\})=\delta m(\{x \in(-1,1):|\phi(x)|>\lambda\}), \quad \lambda>0 .
$$

Consider the dilation operator $E_{\delta}: \tilde{X} \rightarrow \tilde{X}$ for $\delta<1$ given above, that is, $E_{\delta}(f)(t)=$ $f(\delta t)$ for $0 \leqslant s \leqslant \min \{2,1 / \delta\}$ and zero otherwise. For the decreasing rearrangements $\phi^{*}$ and $\varphi^{*}$ of $\phi$ and $\varphi$, respectively, it follows from (10) that

$$
\phi^{*}=E_{\delta}\left(\varphi^{*}\right) .
$$

Consequently, with $\left\|E_{\delta}\right\|$ denoting the operator norm of $E_{\delta}: \tilde{X} \rightarrow \tilde{X}$, we have

$$
\|\phi\|_{X}=\left\|\phi^{*}\right\|_{\tilde{X}}=\left\|E_{\delta}\left(\varphi^{*}\right)\right\|_{\tilde{X}} \leqslant\left\|E_{\delta}\right\| \cdot\left\|\varphi^{*}\right\|_{\tilde{X}}=\left\|E_{\delta}\right\| \cdot\|\varphi\|_{X} .
$$

It follows, from (2), (5) , (9) and (11) that

$$
\begin{aligned}
\|\phi\|_{X} & \leqslant\left\|E_{\delta}\right\| \cdot\|\varphi\|_{X} \\
& \leqslant\left\|E_{\delta}\right\| \cdot\left\|\left(\sum_{n=1}^{N}\left|a_{n}\right|^{2}\left|T\left(\chi_{A_{n}}\right)\right|^{2}\right)^{1 / 2}\right\|_{X} \\
& \leqslant \sqrt{2}\left\|E_{\delta}\right\| \cdot\|\phi\|_{[T, X]} .
\end{aligned}
$$


That is, there exists a constant $M>0$, depending exclusively on $X$, such that

$$
M\|\phi\|_{X} \leqslant\|\phi\|_{[T, X]},
$$

for all simple functions $\phi$.

In order to extend (12) to all functions in $[T, X]$ fix $f \in[T, X]$. For every simple function $\phi$ satisfying $|\phi| \leqslant|f|$ it follows from (12) that

$$
M\|\phi\|_{X} \leqslant\|\phi\|_{[T, X]} \leqslant\|f\|_{[T, X]} .
$$

Taking the supremum with respect to all such $\phi$ yields, via the Fatou property of $X$, that $f \in X$ and

$$
M\|f\|_{X} \leqslant\|f\|_{[T, X]} .
$$

In particular, $[T, X] \subseteq X$. Consequently, $[T, X]=X$ with equivalent norms. Thus, no genuine $X$-valued extension of $T_{X}: X \rightarrow X$ is possible.

The above Theorem has an immediate consequence. Namely, it extends, to all r.i. spaces with non-trivial Boyd indices, certain results known for those r.i. spaces $X$ satisfying $0<\underline{\alpha}_{X} \leqslant \bar{\alpha}_{X}<1 / 2$ or $1 / 2<\underline{\alpha}_{X} \leqslant \bar{\alpha}_{X}<1$, [4, Corollary 4.8], and for $X=L^{2}(-1,1)$, [4, Corollaries 5.4 and 5.5].

Corollary. Let $X$ be a r.i. space on $(-1,1)$ with non-trivial Boyd indices.

(a) There exists a constant $\beta>0$ such that, for every $f \in X$, we have

$$
\frac{\beta}{4}\|f\|_{X} \leqslant \sup _{A \in \mathcal{B}}\left\|T_{X}\left(\chi_{A} f\right)\right\|_{X} \leqslant \sup _{|\theta|=1}\left\|T_{X}(\theta f)\right\|_{X} \leqslant \sup _{|h| \leqslant|f|}\left\|T_{X}(h)\right\|_{X} \leqslant\|f\|_{X} .
$$

(b) For a function $f \in L^{1}$ the following conditions are equivalent.

(a) $f \in X$.

(b) $T\left(f \chi_{A}\right) \in X$ for every $A \in \mathcal{B}$.

(c) $T(f \theta) \in X$ for every $\theta \in L^{\infty}$ with $|\theta|=1$ a.e.

(d) $T(h) \in X$ for every $h \in L^{0}$ with $|h| \leqslant|f|$ a.e.

Acknowledgement. We are grateful to the referee for some valuable suggestions.

\section{REFERENCES}

[1] C. Bennett, R. Sharpley, Interpolation of Operators, Academic Press, Boston, 1988.

[2] D. W. Boyd, Indices of function spaces and their relationship to interpolation, Can. J. Math. 21 (1969), 1245-1254.

[3] H. K. Cheng, N. Rott, Generalizations of the inversion formula of thin airfoil theory, J. Rational Mech. Anal. 3 (1954), 357-382.

[4] G. P. Curbera, S. Okada, W. J. Ricker, Inversion and extension of the finite Hilbert transform on (-1,1), Ann. Mat. Pura Appl. (4), 198 (2019), 1835-1860.

[5] G. P. Curbera, S. Okada, W. J. Ricker, Extension and integral representation of the finite Hilbert transform in rearrangement invariant spaces, Quaest. Math., 43 (2020), 783-812.

[6] J. Diestel, H. Jarchow, A. Tonge, Absolutely Summing Operators, Cambridge University Press, Cambridge, 1995.

[7] R. Duduchava, Integral Equations in Convolution with Discontinuous Presymbols. Singular Integral Equations with Fixed Singularities, and their Applications to some Problems of Mechanics, Teubner Verlagsgesellschaft, Leipzig, 1979. 
[8] F. D. Gakhov, Boundary Value Problems, Dover Publications, Inc., New York, NY, 1990.

[9] I. Gohberg, N. Krupnik, One-Dimensional Linear Singular Integral Operators Vol. I. Introduction, Operator Theory Advances and Applications 53, Birkhäuser, Berlin, 1992.

[10] I. Gohberg, N. Krupnik, One-Dimensional Linear Singular Integral Operators Vol. II. General Theory and Applications, Operator Theory Advances and Applications 54, Birkhäuser, Berlin, 1992.

[11] A. Katsevich, A. Tovbis, Finite Hilbert transform with incomplete data: null-space and singular values, Inverse Problems 28 (2012), 105006, 28 pp.

[12] F. W. King, Hilbert Transforms Vol. I, Cambridge University Press, Cambridge New York, 2009.

[13] S. G. Krein, Ju. I. Petunin, E. M. Semenov, Interpolation of Linear Operators, Amer. Math. Soc., Providence R. I., 1982.

[14] E. Laeng, On the $L^{p}$ norms of the Hilbert transform of a characteristic function, J. Funct. Anal. 262 (2012), 4534-4539.

[15] S. Mikhlin, S. Prössdorf, Singular Integral Operators, Springer-Verlag, Berlin, 1986.

[16] N. I. Muskhelishvili, Singular Integral Equations, Wolters-Noordhoff Publishing, Groningen, 1967.

[17] S. Okada, D. Elliott, The finite Hilbert transform in $\mathcal{L}^{2}$, Math. Nachr. 153 (1991), 43-56.

[18] S. Okada, W. J. Ricker, E. A. Sánchez-Pérez, Optimal Domain and Integral Extension of Operators: Acting in Function Spaces, Operator Theory Advances and Applications 180, Birkhäuser, Berlin, 2008.

[19] A. D. Polyanin, A. V. Manzhirov, Handbook of Integral Equations, 2nd ed., CRC Press, Boca Raton, FL, 2008.

[20] E. Reissner, Boundary value problems in aerodynamics of lifting surfaces in non-uniform motion, Bull. Amer. Math. Soc. 55 (1949), 825-850.

[21] E. Y. Sidky, Xiaochuan Pan, Recovering a compactly supported function from knowledge of its Hilbert transform on a finite interval, IEEE Signal Processing Letters 12 (2005), 97-100.

[22] H. Söhngen, Zur Theorie der endlichen Hilbert-Transformation, Math. Z. 60 (1954), 31-51.

[23] F. G. Tricomi, On the finite Hilbert transform, Quart. J. Math. 2 (1951), 199-211.

[24] F. G. Tricomi, Integral Equations, Interscience, New York, 1957.

Facultad de Matemáticas \& IMUS, Universidad de Sevilla, Calle Tarfia s/n, Sevilla 41012, Spain

Email address: curbera@us.es

School of Natural Sciences (Maths/ Physics), University of Tasmania, Private Bag 37, Hobart, Tas. 7001, Australia

Email address: susumu.okada@utas.edu.au

Math.-Geogr. Fakultät, Katholische Universität Eichstätt-Ingolstadt, D-85072 Eichstätt, GERMAnY

Email address: werner.ricker@ku.de 